\title{
Some Improvements of Jensen's Inequality via 4-Convexity and Applications
}

\author{
Hidayat Ullah $\mathbb{D}^{1},{ }^{1}$ Muhammad Adil Khan $\mathbb{D}^{1},{ }^{1}$ Tareq Saeed, ${ }^{2}$ \\ and Zaid Mohammed Mohammed Mahdi Sayed $\mathbb{D}^{3}$ \\ ${ }^{1}$ Department of Mathematics, University of Peshawar, Peshawar 25000, Pakistan \\ ${ }^{2}$ Nonlinear Analysis and Applied Mathematics- (NAAM-) Research Group, Department of Mathematics, Faculty of Science, \\ King Abdulaziz University, P.O. Box 80203, Jeddah 21589, Saudi Arabia \\ ${ }^{3}$ Department of Mathematics, University of Sáadah, Sáadah 1872, Yemen
}

Correspondence should be addressed to Zaid Mohammed Mohammed Mahdi Sayed; zaidmohamm56@gmail.com

Received 4 October 2021; Accepted 8 November 2021; Published 17 January 2022

Academic Editor: Sarfraz Nawaz Malik

Copyright (C) 2022 Hidayat Ullah et al. This is an open access article distributed under the Creative Commons Attribution License, which permits unrestricted use, distribution, and reproduction in any medium, provided the original work is properly cited.

The intention of this note is to investigate some new important estimates for the Jensen gap while utilizing a 4-convex function. We use the Jensen inequality and definition of convex function in order to achieve the required estimates for the Jensen gap. We acquire new improvements of the Hölder and Hermite-Hadamard inequalities with the help of the main results. We discuss some interesting relations for quasi-arithmetic and power means as consequences of main results. At last, we give the applications of our main inequalities in the information theory. The approach and techniques used in the present note may simulate more research in this field.

\section{Introduction}

The theory of convex functions performs an extremely significant and consequential role in several areas of pure and applied sciences. Due to its numerous and extensive applications, the concept of convex functions has been extended and generalized in many directions. The most important and elegant aspect of the class of convex functions, which attracted many researchers, is its deep relation with theory of inequalities [1-3]. In the literature, there are several well-known inequalities which are the direct consequences and applications of convexity $[4,5]$. In this respect, some of the noted inequalities associated with the class of convex functions are majorization, Hermite-Hadamard and Jensen-Mercer inequalities [6]. Among these inequalities, one of the considerable and vital inequalities which are studied very widely in the literature is the Jensen inequality. This celebrated inequality reads as follows:
Theorem 1. Assume that I is an interval of real numbers and $\Psi$ is a convex function on I. If $y_{j} \in I$ and $w_{j}>0$ for $j=1,2$, $\cdots, n$ with $W=\sum_{j=1}^{n} w_{j}$, then

$$
\Psi\left(\frac{1}{W} \sum_{j=1}^{n} w_{j} y_{j}\right) \leq \frac{1}{W} \sum_{j=1}^{n} w_{j} \Psi\left(y_{j}\right) .
$$

Inequality (1) will be true in the reverse direction, if the function $\Psi$ is concave on $I$.

The Jensen inequality has multitudinous applications in Mathematics [7-11], Statistics [12], Economics [13] and Information Theory [14], etc. The most interesting and attractive applications of this inequality is that it generalized the classical convexity. Moreover, there are several inequalities which are the direct consequences of this inequality 
such as Ky Fan, Cauchy, Hermite-Hadamard and Hölder inequalities. Due to the vast applications of the Jensen inequality, many researchers dedicated their work to this inequality. This inequality has been extended, improved, and refined in multidirections by using different techniques and principals. For some more extensive literature concerning to the Jensen inequality, see $[15,16]$.

\section{Main Results}

In the present part, we discuss the main results. Let us begin this section with the following theorem, in which we acquire an upper bound for the Jensen gap.

Theorem 2. Assume that $I$ is an interval in $\mathbb{R}, x_{i} \in I$ and $p_{i}>$ 0 for $i=1,2, \cdots, n$ with $P_{n}:=\sum_{i=1}^{n} p_{i}$ and $\bar{x}=1 / P_{n} \sum p_{i} x_{i}$. If $\Psi$ is a twice differentiable function such that $\Psi$ is 4-convex on $I$, then

$\frac{1}{P_{n}} \sum_{i=1}^{n} p_{i} \Psi\left(x_{i}\right)-\Psi(\bar{x}) \leq \frac{1}{6 P_{n}} \sum_{i=1}^{n} p_{i}\left(\bar{x}-x_{i}\right)^{2}\left(2 \Psi^{\prime \prime}(\bar{x})+\Psi^{\prime \prime}\left(x_{i}\right)\right)$.

Inequality (2) will be true in the opposite direction, if the function $\Psi$ is 4-concave.

Proof. Without misfortune of sweeping statement, assume that $\bar{x} \neq x_{i}$ for $i=1,2, \cdots, n$. Utilizing integration by parts, we have

$$
\begin{aligned}
\frac{1}{P_{n}} \sum_{i=1}^{n} p_{i}\left(\bar{x}-x_{i}\right)^{2} \int_{0}^{1} t \Psi^{\prime \prime}\left(t \bar{x}+(1-t) x_{i}\right) d t \\
=\frac{1}{P_{n}} \sum_{i=1}^{n} p_{i}\left(\bar{x}-x_{i}\right)^{2}\left(\left.\frac{t}{\bar{x}-x_{i}} \Psi^{\prime}\left(t \bar{x}+(1-t) x_{i}\right)\right|_{0} ^{1}\right. \\
\left.\quad-\frac{1}{\bar{x}-x_{i}} \int_{0}^{1} \Psi^{\prime}\left(t \bar{x}+(1-t) x_{i}\right) d t\right) \\
=\frac{1}{P_{n}} \sum_{i=1}^{n} p_{i}\left(\bar{x}-x_{i}\right)^{2}\left(\frac{\Psi^{\prime}(\bar{x})}{\bar{x}-x_{i}}-\left.\frac{t}{\left(\bar{x}-x_{i}\right)^{2}} \Psi\left(t \bar{x}+(1-t) x_{i}\right)\right|_{0} ^{1}\right) \\
=\frac{1}{P_{n}} \sum_{i=1}^{n} p_{i}\left(\bar{x}-x_{i}\right)^{2}\left(\frac{\Psi^{\prime}(\bar{x})}{\bar{x}-x_{i}}-\frac{t}{\left(\bar{x}-x_{i}\right)^{2}}\left(\Psi(\bar{x})-\Psi\left(x_{i}\right)\right)\right) \\
=\frac{1}{P_{n}} \sum_{i=1}^{n} p_{i}\left(\bar{x}-x_{i}\right) \Psi^{\prime}(\bar{x})-\frac{1}{P_{n}} \sum_{i=1}^{n} p_{i}\left(\Psi(\bar{x})-\Psi\left(x_{i}\right)\right) \\
=\frac{1}{P_{n}} \sum_{i=1}^{n} p_{i} \Psi\left(x_{i}\right)-\Psi(\bar{x}),
\end{aligned}
$$

which implies that

$\frac{1}{P_{n}} \sum_{i=1}^{n} p_{i} \Psi\left(x_{i}\right)-\Psi(\bar{x})=\frac{1}{P_{n}} \sum_{i=1}^{n} p_{i}\left(x_{i}-\bar{x}\right)^{2} \int_{0}^{1} t \Psi^{\prime \prime}\left(t \bar{x}+(1-t) x_{i}\right) d t$.

Since, the function $\Psi$ is 4-convex on $I$. Therefore, using the definition of convex function on the right hand side of
(4), we receive

$$
\begin{aligned}
& \frac{1}{P_{n}} \sum_{i=1}^{n} p_{i} \Psi\left(x_{i}\right)-\Psi(\bar{x}) \\
& \quad \leq \frac{1}{P_{n}} \sum_{i=1}^{n} p_{i}\left(x_{i}-\bar{x}\right)^{2} \int_{0}^{1}\left(t^{2} \Psi^{\prime \prime}(\bar{x})+t(1-t) \Psi^{\prime \prime}\left(x_{i}\right)\right) d t \\
& \quad=\frac{1}{P_{n}} \sum_{i=1}^{n} p_{i}\left(\bar{x}-x_{i}\right)^{2}\left(\Psi^{\prime \prime}(\bar{x}) \int_{0}^{1} t^{2} d t+\Psi^{\prime \prime}\left(x_{i}\right) \int_{0}^{1}\left(t-t^{2}\right) d t\right) \\
& \quad=\frac{1}{P_{n}} \sum_{i=1}^{n} p_{i}\left(\bar{x}-x_{i}\right)^{2}\left(\frac{1}{3} \Psi^{\prime \prime}(\bar{x})+\frac{1}{6} \Psi^{\prime \prime}\left(x_{i}\right)\right),
\end{aligned}
$$

which is equivalent to (2).

The integral version of (2) is stated in the following theorem.

Theorem 3. Assume that $I$ is an interval in $\mathbb{R}, \Psi: I \longrightarrow \mathbb{R}$ that is a twice differentiable function such that $\Psi$ is 4convex and $\phi, \varphi:[a, b] \longrightarrow I$ are integrable functions with $\varphi$ $\geq 0$ on $[a, b]$. Also, assume that $\Psi \circ \phi:[a, b] \longrightarrow \mathbb{R}$ is an integrable function, $\bar{\varphi}:=\int_{a}^{b} \varphi(x) d x>0$ and $\bar{\phi}:=\frac{1}{\varphi} \int_{a}^{b} \varphi(x) \phi(x) d x$. Then

$$
\begin{aligned}
& \frac{1}{\varphi} \int_{a}^{b} \varphi(x) \Psi \circ \phi(x) d x-\Psi(\bar{\phi}) \\
& \quad \leq \frac{1}{6 \bar{\varphi}} \int_{a}^{b} \varphi(x)(\bar{\phi}-\phi(x))^{2}\left(2 \Psi^{\prime \prime}(\bar{\phi})+\Psi^{\prime \prime}(\phi(x))\right) d x
\end{aligned}
$$

Inequality (6) will be true in the reverse sense, if $\Psi$ is a 4concave function.

In the next theorem, we acquire a lower bound for the Jensen gap while utilizing the Jensen inequality.

Theorem 4. Assume that all the suppositions of Theorem 2 are valid, then

$$
\frac{1}{P_{n}} \sum_{i=1}^{n} p_{i} \Psi\left(x_{i}\right)-\Psi(\bar{x}) \geq \frac{1}{2 P_{n}} \sum_{i=1}^{n} p_{i}\left(\bar{x}-x_{i}\right)^{2} \Psi^{\prime \prime}\left(\frac{2 \bar{x}+x_{i}}{3}\right) .
$$

Inequality (7) will become true in the reverse direction, if the function $\Psi$ is 4-concave.

Proof. Since, the function $\Psi$ is 4-convex on $I$. Therefore, applying integral Jensen's inequality on the right of (4), we get 


$$
\begin{aligned}
& \frac{1}{P_{n}} \sum_{i=1}^{n} p_{i} \Psi\left(x_{i}\right) \Psi(\bar{x}) \\
& \quad=\frac{1}{P_{n}} \sum_{i=1}^{n} p_{i}\left(\bar{x}-x_{i}\right)^{2}\left(\frac{\int_{0}^{1} t \Psi^{\prime \prime}\left(t \bar{x}+(1-t) x_{i}\right) d t}{\int_{0}^{1} t d t}\right) \\
& \quad \geq \frac{1}{2 P_{n}} \sum_{i=1}^{n} p_{i}\left(\bar{x}-x_{i}\right)^{2} \Psi^{\prime \prime}\left(\frac{\int_{0}^{1} t\left(t \bar{x}+(1-t) x_{i}\right) d t}{\int_{0}^{1} t d t}\right) \\
& \quad=\frac{1}{2 P_{n}} \sum_{i=1}^{n} p_{i}\left(\bar{x}-x_{i}\right)^{2} \Psi^{\prime \prime}\left(\frac{\bar{x} \int_{0}^{1} t^{2} d t+x_{i} \int_{0}^{1}\left(t-t^{2}\right) d t}{\int_{0}^{1} t d t}\right) \\
& \quad=\frac{1}{2 P_{n}} \sum_{i=1}^{n} p_{i}\left(\bar{x}-x_{i}\right)^{2} \Psi^{\prime \prime}\left(\frac{2 \bar{x}+x_{i}}{3}\right),
\end{aligned}
$$

which is the required inequality.

The analogous inequality of (7) is given in the following theorem.

Theorem 5. Suppose that all the hypotheses of Theorem 3 are true. Then

$$
\begin{aligned}
& \frac{1}{\overline{\bar{\varphi}}} \int_{a}^{b} \varphi(x) \Psi \circ \phi(x) d x-\Psi(\bar{\phi}) \\
& \quad \geq \frac{1}{2 \bar{\varphi}} \int_{a}^{b} \varphi(x)(\bar{\phi}-\phi(x))^{2} \Psi^{\prime \prime}\left(\frac{2 \bar{\phi}+\phi(x)}{3}\right) d x .
\end{aligned}
$$

If the function $\Psi$ is 4-concave, then the inequality holds in the opposite direction.

\section{Numerical Experiments}

In this section, we are going to provide some simple examples to show how sharp our estimates for the Jensen gap.

Example 6. Consider the functions $\Psi(x)=x^{4}, \phi(x)=x$ and $\varphi(x)=1$ for all $x \in[0,1]$. Then, $\Psi^{\prime \prime}(x)=12 x^{2} \geq 0$ and $\Psi^{\prime \prime \prime \prime}(x)$ $=24>0$ on $[0,1]$. This verifies that the function $\Psi$ is convex as well as 4 -convex. Now, utilizing (6) for $\Psi(x)=x^{4}, \phi(x)$ $=x, \varphi(x)=1, a=0$, and $b=1$, we get

$$
0.1375<0.15
$$

Using above functions with the given interval in inequality (4) in [17], we acquire

$$
0.1375<0.25
$$

From inequalities (10) and (11) it is clear that the bounds given in (6) provide a good and better estimate for the Jensen gap. Moreover, the inequality (10) shows that the value of the obtained estimate for the Jensen gap given in (6) is very close to the value of the Jensen gap.
Example 7. Consider the functions $\Psi(x)=(1-x)^{5}, \varphi(x)=1$, and $\phi(x)=1$ for all $x \in[0,1]$. Then, $\Psi^{\prime \prime}(x)=20(1-x)^{3}$ and $\Psi^{\prime \prime \prime \prime}(x)=120(1-x)$. Clearly, both $\Psi^{\prime \prime}$ and $\Psi^{\prime \prime \prime}$ are nonnegative on $[0,1]$. This shows that the function $\Psi(x)=(1-x)^{5}$ is convex as well as 4-convex. Utilizing $\Psi(x)=(1-x)^{5}, \Psi^{\prime \prime}$ $(x)=20(1-x)^{3}, \varphi(x)=1$ and $\phi(x)=1$ in (6), we obtain

$$
0.1345<0.1666 \text {. }
$$

Now, using the chosen functions in the inequality (4) in [17], we acquire

$$
0.1345<0.4166 \text {. }
$$

From (12) and (13) it is clear that the inequality (6) provides an efficient and superior estimate as compared to the inequality (4) in [17].

Example 8. Assume that the functions $\Psi(x)=\exp x, \varphi(x)=1$ , and $\phi(x)=x$ are defined on $[0,1]$. Then, $\Psi^{\prime \prime}(x)=\exp x$ and $\Psi^{\prime \prime \prime \prime}(x)=\exp x$. Obviously, both the functions $\Psi^{\prime \prime}$ and $\Psi^{\prime \prime \prime \prime}$ are nonnegative on $\in[0,1]$. This confirms the convexity and 4-convexity of the function $\Psi(x)=\exp x$. Choosing $\Psi(x)=\exp x, \Psi^{\prime \prime}(x)=\exp x, \varphi(x)=1$ and $\phi(x)=x$ in (6), we obtain

$$
0.0695<0.0704 \text {. }
$$

Now, using the given functions in the inequality (4) in [17], we acquire

$$
0.0695<0.0996 \text {. }
$$

Again, from (14) and (15), it is obvious that the estimate provided by inequality (6) for the Jensen gap is better than the estimate provided by inequality (4) in [17]. Moreover, the value of the estimate for the Jensen gap in (6) is very close to the value of the Jensen gap.

Remark 9. The authors in [17] compared the value of estimate for the Jensen gap in the inequality (4) with the value of the estimates for the Jensen gap in inequalities (5) and (8) in [18]. From the comparison, the authors declared that the estimate for the Jensen gap in inequality (4) in [17] is better than the estimates for the Jensen gap in inequalities (5) and (8) in [18]. Hence from this, we can also conclude that our estimate for the Jensen gap may be better than the estimates for the Jensen gap in (5) and (8) in [18].

\section{Applications for Classical Inequalities}

This section is devoted to the consequences of main results. In this section, we obtain some improvements for the Hölder and Hermite-Hadamard inequalities with the help of our main results. Furthermore, we acquire different relations for the power and quasi-arithmetic means with the utilization of our obtained results. 
In the following proposition, we give an improvement for the Hölder inequality with the help of Theorem 2.

Proposition 10. Let $m_{1}=\left(\zeta_{1}, \zeta_{2}, \cdots, \zeta_{n}\right)$ and $m_{2}=\left(\gamma_{1}, \gamma_{2}, \cdots\right.$, $\left.\gamma_{n}\right)$ be two positive $n$-tuples and $p, q>1$, such that $p \notin(2,3)$ . If $1 / p+1 / q=1$, then

$$
\begin{aligned}
& \left(\sum_{i=1}^{n} \zeta_{i}^{p}\right)^{1 / p}\left(\sum_{i=1}^{n} \gamma_{i}^{q}\right)^{1 / q}-\sum_{i=1}^{n} \gamma_{i} \zeta_{i} \\
& \leq\left[\frac{p(p-1)}{6} \sum_{i=1}^{n} \gamma_{i}^{q}\left(\frac{\sum_{i=1}^{n} \gamma_{i} \zeta_{i}}{\sum_{i=1}^{n} \gamma_{i}^{q}}-\zeta_{i} \gamma_{i}^{-q / p}\right)^{2}\right. \\
& \left.\quad \times\left(\frac{\sum_{i=1}^{n} \gamma_{i} \zeta_{i}}{\sum_{i=1}^{n} \gamma_{i}^{q}}-\zeta_{i} \gamma_{i}^{-q / p}\right)\right]^{1 / p}\left(\sum_{i=1}^{n} \gamma_{i}^{q}\right)^{\frac{1}{q}}
\end{aligned}
$$

Proof. Since the function $\Psi=x^{p}$ is convex as well as 4convex on $(0, \infty)$ for all $p>1, p \notin(2,3)$. Therefore, utilizing (2) by choosing $\Psi(x)=x^{p}$ and $p_{i}=\gamma_{i}^{q}, x_{i}=\zeta_{i} \gamma_{i}^{-q / p}$ for all $i$ $\in\{1,2, \cdots, n\}$ and then taking power $1 / p$, we get

$$
\begin{gathered}
\left(\left(\sum_{i=1}^{n} \zeta_{i}^{p}\right)\left(\sum_{i=1}^{n} \gamma_{i}^{q}\right)^{p-1}-\left(\sum_{i=1}^{n} \gamma_{i} \zeta_{i}\right)^{p}\right)^{1 / p} \\
\leq\left[\frac{p(p-1)}{6} \sum_{i=1}^{n} \gamma_{i}^{q}\left(\frac{\sum_{i=1}^{n} \gamma_{i} \zeta_{i}}{\sum_{i=1}^{n} \gamma_{i}^{q}}-\zeta_{i} \gamma_{i}^{-(q / p)}\right)^{2}\right. \\
\left.\quad \times\left(\frac{\sum_{i=1}^{n} \gamma_{i} \zeta_{i}}{\sum_{i=1}^{n} \gamma_{i}^{q}}-\zeta_{i} \gamma_{i}^{-(q / p)}\right)\right]^{1 / p}\left(\sum_{i=1}^{n} \gamma_{i}^{q}\right)^{1 / q} .
\end{gathered}
$$

As the inequality

$$
a^{l}-b^{l} \leq(a-b)^{l}
$$

holds, for all $a, b \geq 0$ and $l \in[0,1]$, thus using (18) for $a=($ $\left.\sum_{i=1}^{n} \zeta_{i}^{p}\right)\left(\sum_{i=1}^{n} \gamma_{i}^{q}\right)^{p-1}, b=\left(\sum_{i=1}^{n} \gamma_{i} \zeta_{i}\right)^{p}$ and $l=1 / p$, we obtain

$$
\begin{aligned}
& \left(\sum_{i=1}^{n} \zeta_{i}^{p}\right)^{1 / l}\left(\sum_{i=1}^{n} \gamma_{i}^{q}\right)^{1 / q}-\sum_{i=1}^{n} \gamma_{i} \zeta_{i} \\
& \leq\left(\left(\sum_{i=1}^{n} \zeta_{i}^{p}\right)\left(\sum_{i=1}^{n} \gamma_{i}^{q}\right)^{p-1}-\left(\sum_{i=1}^{n} \gamma_{i} \zeta_{i}\right)\right)^{1 / p} .
\end{aligned}
$$

Now, comparing (17) and (19), we acquire (16).

Another consequence of Theorem 2 is given in the following corollary, in which we provide a relation for the Hölder inequality.

Corollary 11. Let $m_{1}=\left(\zeta_{1}, \zeta_{2}, \cdots, \zeta_{n}\right)$ and $m_{2}=\left(\gamma_{1}, \gamma_{2}, \cdots, \gamma_{n}\right)$ be two positive n-tuples, $0<p<1$ and $q=p /(p-1)$ such that $1 / p \notin(2,3)$. If $1 / p+1 / q=1$, then

$$
\begin{aligned}
& \sum_{i=1}^{n} \gamma_{i} \zeta_{i}-\left(\sum_{i=1}^{n} \zeta_{i}^{p}\right)^{1 / p}\left(\sum_{i=1}^{n} \gamma_{i}^{q}\right)^{1 / q} \\
& \quad \leq \frac{1-p}{6 p^{2}} \sum_{i=1}^{n} \gamma_{i}^{q}\left(\frac{\sum_{i=1}^{n} \zeta_{i}^{p}}{\sum_{i=1}^{n} \gamma_{i}^{q}}-\zeta_{i}^{p} \gamma_{i}^{-q}\right)^{2} \times\left(\frac{\sum_{i=1}^{n} \zeta_{i}^{p}}{\sum_{i=1}^{n} \gamma_{i}^{q}}-\zeta_{i}^{p} \gamma_{i}^{-q}\right)
\end{aligned}
$$

Proof. For $p \in(0,1)$ such that $1 / p \notin(2,3)$, the function $\Psi(x$ )$=x^{1 / p}$ is convex as well as 4 -convex on $[0, \infty]$. Therefore, utilizing (2) by choosing $\Psi(x)=x^{1 / p}, p_{i}=\gamma_{i}^{q}$, and $x_{i}=\gamma_{i}^{-q}$ $\zeta_{i}^{p}$, we get (20).

As a consequence of Theorem 4, we acquire another improvement for the Hölder inequality which is stated in the following corollary.

Corollary 12. Assume that $m_{1}=\left(\gamma_{1}, \gamma_{2}, \cdots, \gamma_{n}\right)$ and $m_{2}=\left(\zeta_{1}\right.$ $\left., \zeta_{2}, \cdots, \zeta_{n}\right)$ are two $n$-tuples such that $\gamma_{i}, \zeta_{i}>0$ for all $i \in\{1$, $2, \cdots, n\}$. If $p \in(0,1)$ and $q=p /(p-1)$ such that $1 / p \notin(2,3)$, then

$$
\begin{aligned}
\sum_{i=1}^{n} \gamma_{i} \zeta_{i}-\left(\sum_{i=1}^{n} \zeta_{i}^{p}\right)^{1 / p}\left(\sum_{i=1}^{n} \gamma_{i}^{q}\right)^{1 / q} \\
\geq \frac{1-p}{2 p^{2}} \sum_{i=1}^{n} \gamma_{i}^{q}\left(\frac{\sum_{i=1}^{n} \zeta_{i}^{p}}{\sum_{i=1}^{n} \gamma_{i}^{q}}-\zeta_{i}^{p} \gamma_{i}^{-q}\right)^{2} \\
\times\left(\frac{2 \sum_{i=1}^{n} \zeta_{i}^{p}+\zeta_{i}^{p} \gamma_{i}^{-q} \sum_{i=1}^{n} \gamma_{i}^{q}}{3 \sum_{i=1}^{n} \gamma_{i}^{q}}\right)
\end{aligned}
$$

Proof. The function $\Psi(x)=x^{1 / p}$ is both convex and 4-convex for $x \geq 0$ with $p \in(0,1)$ such that $1 / p \notin(2,3)$. Therefore, inequality (21) can easily be acquired by putting $\Psi(x)=$ $x^{1 / p}, p_{i}=\gamma_{i}^{q}$, and $x_{i}=\gamma_{i}^{-q} \zeta_{i}^{p}$ in (7).

Now, we recall the definition of power mean.

Definition 13. Let $m_{1}=\left(\gamma_{1}, \gamma_{2}, \cdots, \gamma_{n}\right)$ and $m_{2}=\left(\zeta_{1}, \zeta_{2}, \cdots, \zeta_{n}\right)$ be two $n$-tuples such that $\gamma_{i}, \zeta_{i} \in(0, \infty)$ for all $i \in\{1,2, \cdots, n\}$ with $\bar{\gamma}=\sum_{i=1}^{n} \gamma_{i}$. Then, the power mean of order $p \in \mathbb{R}$ is defined by

$$
M_{p}\left(m_{1}, m_{2}\right)= \begin{cases}\left(\frac{1}{\gamma} \sum_{i=1}^{n} \gamma_{i} \zeta_{i}^{p}\right)^{1 / p}, & p \neq 0 \\ \left(\prod_{i=1}^{n} \zeta_{i}^{\gamma_{i}}\right)^{\frac{1}{\gamma}}, & p=0 .\end{cases}
$$

As a consequence of Theorem 2, in the following corollary, we give bound for the power mean.

Corollary 14. Let $m_{1}=\left(\gamma_{1}, \gamma_{2}, \cdots, \gamma_{n}\right)$ and $m_{2}=\left(\zeta_{1}, \zeta_{2}, \cdots, \zeta_{n}\right)$ be two positive n-tuples such that $\bar{\gamma}=\sum_{i=1}^{n} \gamma_{i}$ and $r$ and $t$ be 
nonzero real numbers. Then the following statements are true:

(i) If $r>0$ with $t \geq 3 r$ or $2 r \geq t \geq r$ or $0>t$, then

$$
\begin{aligned}
M_{r}^{r}\left(m_{1}, m_{2}\right)- & M_{r}^{t}\left(m_{1}, m_{2}\right) \leq \frac{t(t-r)}{6 r^{2} \bar{\gamma}} \sum_{i=1}^{n} \gamma_{i}\left(M_{r}^{r}\left(m_{1}, m_{2}\right)-\zeta_{i}^{r}\right)^{2} \\
& \times\left(M_{r}^{t-2 r}\left(m_{1}, m_{2}\right)+\zeta_{i}^{t-2}\right) .
\end{aligned}
$$

(ii) If $r<0$ with $t \leq 3 r$ or $2 r \leq t \leq r$ or $0<t$, then (23) holds.

(iii) If $r>0$ with $3 r>t>2 r$ or $r<0$ with $3 r<t<2 r$, then (23) holds in the reverse direction.

Proof.

(i) For $x>0$, the function $\Psi(x)=x^{t / r}$ is 4 -convex with the given conditions.

Therefore, using (2) by taking $\Psi(x)=x^{t / r}, p_{i}=\gamma_{i}$, and $x_{i}$ $=\zeta_{i}^{r}$, we obtain $(23)$

(ii) If the given conditions are hold, then the function $\Psi(x)=x^{t / r}$ will be 4 -convex on $(0, \infty)$. Thus, (23) can easily be obtained by adopting the procedure of (i)

(iii) The function $\Psi(x)=x^{t / r}$ is 4 -concave on $(0, \infty)$ for the given values of $r$ and $t$. Therefore, we can get the reverse inequality in (23) by adopting the procedure of $(i)$.

In the following result, we present an application of Theorem 4.

Corollary 15. Let $m_{1}, m_{2}$, and $\bar{\gamma}$ be the same as that of Corollary 14 and $r, t \in \mathbb{R}-\{0\}$. Then

(A) If the conditions given in (i) and (ii) are satisfied, then

$$
\begin{aligned}
M_{t}^{t}\left(m_{1}, m_{2}\right)-M_{r}^{t}\left(m_{1}, m_{2}\right) \\
\geq \frac{t(t-r)}{2 \bar{\gamma} r^{2}} \sum_{i=1}^{n} \gamma_{i}\left(M_{r}^{r}\left(m_{1}, m_{2}\right)-\zeta_{i}^{r}\right)^{2} \\
\quad\left(\frac{2 M_{r}^{r}\left(m_{1}, m_{2}\right)+\zeta_{i}^{t-2}}{3}\right)^{t / r-2} .
\end{aligned}
$$

(B) If the conditions in (iii) are fulfilled, then (24) holds in the reverse direction.
Proof.

(A) Since, the function $\Psi(x)=x^{t / r}$ is 4 -convex on $(0, \infty)$ for the conditions given in (i) and (ii) of Corollary 14. Therefore, using (7) for $\Psi(x)=x^{t / r}, p_{i}=\gamma_{i}$, and $x_{i}=\zeta_{i}^{r}$, we obtain (24)

(B) If the condition on $r$ and $t$ mentioned in (iii) of Corollary 14 is true, then the function $\Psi(x)=x^{t / r}$ will be 4-concave for $x>0$. Thus, utilizing (7) while choosing $\Psi(x)=x^{t / r}, p_{i}=\gamma_{i}$, and $x_{i}=\zeta_{i}^{r}$, we obtain the reverse inequality in (24).

In the following corollary, we obtain an interesting relation for different means as a consequence of Theorem 2 .

Corollary 16. Let $m_{1}=\left(\gamma_{1}, \gamma_{2}, \cdots, \gamma_{n}\right)$ and $m_{2}=\left(\zeta_{1}, \zeta_{2}, \cdots, \zeta_{n}\right)$ be positive $n$-tuples with $\bar{\gamma}=\sum_{i=1}^{n} \gamma_{i}$. Then

$$
\frac{M_{1}\left(m_{1}, m_{2}\right)}{M_{0}\left(m_{1}, m_{2}\right)} \leq \exp \left[\frac{1}{6 \bar{\gamma}} \sum_{i=1}^{n} \gamma_{i}\left(M_{1}\left(m_{1}, m_{2}\right)-\zeta_{i}\right)^{2}\left(M_{1}^{-2}\left(m_{1}, m_{2}\right)+\zeta_{i}^{-2}\right)\right] \text {. }
$$

Proof. Let $\Psi(x)=-\ln x, x>0$. Then, $\Psi^{\prime \prime}(x)=1 / x^{2}$ and $\Psi^{\prime \prime}($ $x)=6 / x^{4}$. Clearly, both $\Psi^{\prime \prime}(x)$ and $\Psi^{\prime \prime \prime}(x)$ are positive for all $x>0$. This confirms that the function $\Psi(x)$ is convex as well as 4 -convex on $(0, \infty)$. Therefore, putting $\Psi(x)=-\ln$ $x, p_{i}=\gamma_{i}$, and $x_{i}=\zeta_{i}$ in (2), we acquire (25).

In the following corollary, a relation for distinct means is obtain with the help of Theorem 2 .

Corollary 17. Let hypotheses of Corollary 16 hold. Then

$$
\begin{aligned}
& M_{t}^{t}\left(m_{1}, m_{2}\right)-M_{0}\left(m_{1}, m_{2}\right) \\
& \quad \leq \frac{1}{6 \bar{\gamma}} \sum_{i=1}^{n} \gamma_{i}\left(M_{1}\left(m_{1}, m_{2}\right)-\operatorname{In} \zeta_{i}\right)^{2}\left(2 M_{0}\left(m_{1}, m_{2}\right)+\zeta_{i}\right) .
\end{aligned}
$$

Proof. Consider function $\Psi(x)=\exp x, x \in \mathbb{R}$. Then clearly, $\Psi^{\prime \prime}(x)=\exp x>0$ and $\Psi^{\prime \prime \prime}(x)=\exp x>0$. This shows that the given function is convex as well as 4-convex. Thus, applying (2) by choosing $p_{i}=\gamma_{i}, x_{i}=\ln \zeta_{i}$, and $\Psi(x)=\exp$ $x$, we get (26).

An application of Theorem 4 is acquired in the below corollary.

Corollary 18. Suppose that all the assumptions of Corollary 16 are true, then

$$
\frac{M_{1}\left(m_{1}, m_{2}\right)}{M_{0}\left(m_{1}, m_{2}\right)} \geq \exp \left[\frac{1}{2 \bar{\gamma}} \sum_{i=1}^{n} \gamma_{i}\left(M_{1}\left(m_{1}, m_{2}\right)-\zeta_{i}\right)^{2}\left(\frac{3}{2 M_{1}\left(m_{1}, m_{2}\right)+\zeta_{i}}\right)^{2}\right]
$$


Proof. Put $\Psi(x)=-\ln x, p_{i}=\gamma_{i}$, and $x_{i}=\zeta_{i}$ in (7), we get (27).

The following is another relation for distinct means which is the consequence of Theorem 4 .

Corollary 19. Let the hypotheses of Corollary 16 be fulfilled. Then

$$
\begin{aligned}
& M_{1}\left(m_{1}, m_{2}\right)-M_{0}\left(m_{1}, m_{2}\right) \\
& \geq \frac{1}{2 \bar{\gamma}} \sum_{i=1}^{n} \gamma_{i}\left(M_{0}\left(m_{1}, m_{2}\right)-\operatorname{In} \zeta_{i}\right)^{2} \exp \\
& \quad \cdot\left(\frac{2 M_{0}\left(m_{1}, m_{2}\right)+\operatorname{In} \zeta_{i}}{3}\right) .
\end{aligned}
$$

Proof. Utilizing (7) for $p_{i}=\gamma_{i}, x_{i}=\ln \zeta_{i}$, and $\Psi(x)=\exp x$, we get (28).

Now, we give the definition of quasiarithmetic mean.

Definition 20. Let $m_{1}=\left(\gamma_{1}, \gamma_{2}, \cdots, \gamma_{n}\right)$ and $m_{2}=\left(\zeta_{1}, \zeta_{2}, \cdots, \zeta_{n}\right)$ be positive $n$-tuples with $\bar{\gamma}=\sum_{i=1}^{n} \gamma_{i}$ and $\varphi$ be strictly monotonic continuous function. Then the quasi-arithmetic mean is defined as

$$
M_{\varphi}\left(m_{1}, m_{2}\right)=\varphi^{-1}\left(\frac{1}{\gamma} \sum_{i=1}^{n} \gamma_{i} \varphi\left(\zeta_{i}\right)\right) .
$$

In the following corollary, we obtain a relation for the quasi-arithmetic mean with the help of Theorem 2 .

Corollary 21. Let $m_{1}=\left(\gamma_{1}, \gamma_{2}, \cdots, \gamma_{n}\right)$ and $m_{2}=\left(\zeta_{1}, \zeta_{2}, \cdots, \zeta_{n}\right)$ be positive $n$-tuples with $\bar{\gamma}=\sum_{i=1}^{n} \gamma_{i}$. Also, let $\varphi$ be strictly monotonic continuous function and $\Psi^{\circ} \varphi^{-1}$ be 4-convex on $(0, \infty)$. Then

$$
\begin{aligned}
\frac{1}{\gamma} \sum_{i=1}^{n} \gamma_{i} \Psi\left(\zeta_{i}\right)-\Psi\left(M_{\varphi}\left(m_{1}, m_{2}\right)\right) \\
\leq \frac{1}{6 \bar{\gamma}} \sum_{i=1}^{n} \gamma_{i}\left(\varphi\left(M_{\varphi}\left(m_{1}, m_{2}\right)\right)-\varphi\left(\zeta_{i}\right)\right)^{2} \\
\quad \times\left(2\left(\Psi \circ \varphi^{-1}\right)^{\prime \prime}\left(\varphi\left(M_{\varphi}\left(m_{1}, m_{2}\right)\right)\right)+\left(\Psi \circ \varphi^{-1}\right)^{\prime \prime} \varphi\left(\zeta_{i}\right)\right) .
\end{aligned}
$$

Proof. Since, the function $\Psi \circ \varphi^{-1}$ is 4-convex on $(0, \infty)$. Therefore, choosing $\Psi=\Psi \circ \varphi^{-1}, p_{i}=\gamma_{i}$, and $x_{i}=\phi\left(\zeta_{i}\right)$ in (2), we obtain (30).

As an application of Theorem 4, in the following corollary, we present a relation for the quasi-arithmetic mean.
Corollary 22. Let the hypotheses of Corollary 21 hold. Then

$$
\begin{aligned}
\frac{1}{\gamma} \sum_{i=1}^{n} \gamma_{i} \Psi\left(\zeta_{i}\right)-\Psi\left(M_{\varphi}\left(m_{1}, m_{2}\right)\right) \\
\geq \frac{1}{2 \bar{\gamma}} \sum_{i=1}^{n} \gamma_{i}\left(\varphi\left(M_{\varphi}\left(m_{1}, m_{2}\right)\right)-\varphi\left(\zeta_{i}\right)\right)^{2} \\
\quad \times\left(\left(\Psi \circ \varphi^{-1}\right)^{\prime \prime}\left(\frac{2 \varphi\left(M_{\varphi}\left(m_{1}, m_{2}\right)\right)+\varphi\left(\zeta_{i}\right)}{3}\right)\right) .
\end{aligned}
$$

Proof. Using (7) for $\Psi=\Psi \circ \varphi^{-1}, p_{i}=\gamma_{i}$, and $x_{i}=\varphi\left(\zeta_{i}\right)$, we obtain (31).

In the following corollaries, we present some improvements for the Hermite-Hadamard inequalities with the support of our main results.

Corollary 23. Let $\Psi:[a, b] \longrightarrow \mathbb{R}$ be a 4-convex function. Then

$$
\begin{aligned}
& \frac{1}{b-a} \int_{a}^{b} \Psi(x) d x-\Psi\left(\frac{a+b}{2}\right) \\
& \quad \leq \frac{1}{6(b-a)} \int_{a}^{b}\left(\frac{a+b}{2}-x\right)^{2}\left(2 \Psi^{\prime \prime}\left(\frac{a+b}{2}\right)+\Psi^{\prime \prime}(x)\right) d x
\end{aligned}
$$

If the function $\Psi$ is 4 -concave, then the inequality (32) holds in the reverse direction.

Proof. Since, the function $\Psi$ is 4 -convex on $[a, b]$. Therefore, using (6) for $\varphi(x)=1$ and $\phi(x)=x$, we get (32).

Corollary 24. Assume that the function $\Psi:[a, b] \longrightarrow R$ is 4convex, then

$$
\begin{aligned}
& \frac{1}{b-a} \int_{a}^{b} \Psi(x) d x-\Psi\left(\frac{a+b}{2}\right) \\
& \quad \geq \frac{1}{2(b-a)} \int_{a}^{b}\left(\frac{a+b}{2}-x\right)^{2} \Psi^{\prime \prime}\left(\frac{a+b+x}{3}\right) d x .
\end{aligned}
$$

Inequality (33) will be true in the opposite sense, if the function $\Psi$ is 4-concave.

Proof. Inequality (33) can easily be deduced by choosing $\phi($ $x)=x$ and $\varphi(x)=1$ in (9).

Remark 25. The integral version of the above discrete improvements of Hölder and Hermite-Hadamard inequalities and relations for different means can easily be achieved by using Theorems 3 and 5 .

\section{Applications in Information Theory}

In this part of the note, we are going to discuss some applications of the main inequalities in information theory. These 
applications involve some bounds for different divergences, the Bhattacharyya coefficient and the Shannon entropy.

Definition 26. Let $\Phi$ be a real valued function defined on $[a$ $, b] \subset \mathrm{R}$ and $m_{1}=\left(\gamma_{1}, \gamma_{2}, \cdots, \gamma_{n}\right)$ and $m_{2}=\left(\zeta_{1}, \zeta_{2}, \cdots, \zeta_{n}\right)$ be two $n$-tuples such that $\zeta_{i} / \gamma_{i} \in[a, b]$ and $\gamma_{i}>0$ for all $i \in\{1$, $2, \cdots, n\}$. Then the Csisźar divergence is defined by

$$
D_{c}\left(m_{1}, m_{2}\right)=\sum_{i=1}^{n} \gamma_{i} \Phi\left(\frac{\zeta_{i}}{\gamma_{i}}\right)
$$

Theorem 27. Assume that the function $\Phi:[a, b] \longrightarrow R$ is 4convex and $m_{1}=\left(\gamma_{1}, \gamma_{2}, \cdots, \gamma_{n}\right)$ and $m_{2}=\left(\zeta_{1}, \zeta_{2}, \cdots, \zeta_{n}\right)$ are two $n$-tuples such that $\sum_{i=1}^{n} \zeta_{i} l \sum_{i=1}^{n} \gamma_{i}, \zeta_{i} l \gamma_{i} \in[a, b]$ and $\gamma_{i}>0$ for all $i \in\{1,2, \cdots, n\}$, then

$$
\begin{aligned}
& D_{c}\left(m_{1}, m_{2}\right)-\Phi\left(\frac{\sum_{i=1}^{n} \zeta_{i}}{\sum_{i=1}^{n} \gamma_{i}}\right) \sum_{i=1}^{n} \gamma_{i} \\
& \quad \leq \frac{1}{6} \sum_{i=1}^{n} \gamma_{i}\left(\frac{\sum_{i=1}^{n} \zeta_{i}}{\sum_{i=1}^{n} \gamma_{i}}-\frac{\zeta_{i}}{\gamma_{i}}\right)^{2}\left(2 \Phi^{\prime \prime}\left(\frac{\sum_{i=1}^{n} \zeta_{i}}{\sum_{i=1}^{n} \gamma_{i}}\right)+\Phi^{\prime \prime}\left(\frac{\zeta_{i}}{\gamma_{i}}\right)\right) .
\end{aligned}
$$

Proof. All the hypotheses of this theorem are same as that of Theorem 2.

Thus, using (2) by taking $\Psi=\Phi, p_{i}=\gamma_{i} / \sum_{i=1}^{n} \gamma_{i}$, and $x_{i}=$ $\zeta_{i} / \gamma_{i}$, we obtain (35).

Theorem 28. Suppose that all the assumptions of Theorem 27 are satisfied, then

$$
\begin{aligned}
& D_{c}\left(m_{1}-m_{2}\right)-\Phi\left(\frac{\sum_{i=1}^{n} \zeta_{i}}{\sum_{i=1}^{n} \gamma_{i}}\right) \sum_{i=1}^{n} \gamma_{i} \\
& \quad \geq \frac{1}{2} \sum_{i=1}^{n} \gamma_{i}\left(\frac{\sum_{i=1}^{n} \zeta_{i}}{\sum_{i=1}^{n} \gamma_{i}}-\frac{\zeta_{i}}{\gamma_{i}}\right)^{2} \Phi^{\prime \prime}\left(2 \frac{\sum_{i=1}^{n} \zeta_{i}}{3 \sum_{i=1}^{n} \gamma_{i}}+\frac{\zeta_{i}}{3 \gamma_{i}}\right) .
\end{aligned}
$$

Proof. Since, the function $\Phi$ is 4-convex. Therefore, utilizing (7) for $\Psi=\Phi, p_{i}=\gamma_{i} / \sum_{i=1}^{n} \gamma_{i}$, and $x_{i}=\zeta_{i} / \gamma_{i}$, we get (36).

Definition 29. For any $\delta \in[0, \infty)$ such that $\delta \neq 1$ and arbitrary positive probability distributions $m_{1}=\left(\gamma_{1}, \gamma_{2}, \cdots, \gamma_{n}\right)$ and $m_{2}=\left(\zeta_{1}, \zeta_{2}, \cdots, \zeta_{n}\right)$, the Rényi divergence is defined by

$$
D_{r e}\left(m_{1}, m_{2}\right)=\frac{1}{\delta-1} \log \left(\sum_{i=1}^{n} \gamma_{i}^{\delta} \zeta_{i}^{1-\delta}\right) .
$$

Corollary 30. Assume that $m_{1}=\left(\gamma_{1}, \gamma_{2}, \cdots, \gamma_{n}\right)$ and $m_{2}=\left(\zeta_{1}\right.$ $\left., \zeta_{2}, \cdots, \zeta_{n}\right)$ are two positive probability distributions and $\delta>$ 1 , then

$$
\begin{aligned}
D_{r e}\left(m_{1}, m_{2}\right)-\frac{1}{\delta-1} \sum_{i=1}^{n} \gamma_{i}^{\delta} \log \left(\frac{\gamma_{i}}{\zeta_{i}}\right)^{\delta-1} \\
\leq \frac{1}{6} \sum_{i=1}^{n} \gamma_{i}\left(\sum_{i=1}^{n} \gamma_{i}^{\delta} \zeta_{i}^{1-\delta}-\left(\frac{\gamma_{i}}{\zeta_{i}}\right)^{\delta-1}\right)^{2} \\
\quad \times\left(\frac{2}{\delta-1}\left(\sum_{i=1}^{n} \gamma_{i}^{\delta} \zeta_{i}^{1-\delta}\right)^{-2}+\frac{1}{\delta-1}\left(\frac{\gamma_{i}}{\zeta_{i}}\right)^{2-2 \delta}\right) .
\end{aligned}
$$

Proof. Let $\Phi(x)=1 /(\delta-1) \log x, x>0$. Then, $\Phi^{\prime \prime}(x)=1 /((\delta$ $\left.-1) x^{2}\right)$ and $\Phi^{\prime \prime \prime}(x)=6 /\left((\delta-1) x^{4}\right)$. Clearly, $\Phi^{\prime \prime}(x)>0$ and $\Phi^{\prime \prime \prime}(x)>0$ for all $x \in(0, \infty)$. Thus, this verifies that the function $\Phi(x)=1 /(\delta-1) \log x$ is convex as well as 4convex on $(0, \infty)$. Therefore, using (2) for $\Phi(x)=1 /(\delta-1)$ $\log x, p_{i}=\gamma_{i}$, and $x_{i}=\left(\gamma_{i} / \zeta_{i}\right)^{\delta-1}$, we get (38).

Corollary 31. Let the hypotheses of Corollary 30 hold. Then

$$
\begin{gathered}
D_{r e}\left(m_{1}, m_{2}\right)-\frac{1}{\delta-1} \sum_{i=1}^{n} \gamma_{i}^{\delta} \log \left(\frac{\gamma_{i}}{\zeta_{i}}\right)^{\delta-1} \\
\geq \frac{1}{2(\delta-1)} \sum_{i=1}^{n} \gamma_{i}\left(\sum_{i=1}^{n} \gamma_{i}^{\delta} \zeta_{i}^{1-\delta}-\left(\frac{\gamma_{i}}{\zeta_{i}}\right)^{\delta-1}\right)^{2} \\
\times\left(\frac{3}{2 \sum_{i=1}^{n} \gamma_{i}^{\delta} \zeta_{i}^{1-\delta}+\left(\gamma_{i} / \zeta_{i}\right)^{\delta-1}}\right)^{2} .
\end{gathered}
$$

Proof. Using $\Phi(x)=1 /(\delta-1) \log x, p_{i}=\gamma_{i}$ and $x_{i}=\left(\gamma_{i} / \zeta_{i}\right)^{\delta-1}$ in (7), we get (39).

Definition 32. Let $m_{2}=\left(\zeta_{1}, \zeta_{2}, \cdots, \zeta_{n}\right)$ be a probability distribution with positive entries. Then the Shannon entropy is defined by

$$
E_{s}\left(m_{2}\right)=-\sum_{i=1}^{n} \zeta_{i} \log \zeta_{i}
$$

Corollary 33. Suppose that $m_{2}=\left(\gamma_{1}, \gamma_{2}, \cdots, \gamma_{n}\right)$ is a probability distribution such that $\gamma_{i}>0$ for all $i \in\{1,2, \cdots, n\}$, then

$$
\log n-E_{s}\left(m_{2}\right) \leq \frac{1}{6} \sum_{i=1}^{n} \gamma_{i}\left(n-\frac{1}{\gamma_{i}}\right)^{2}\left(\frac{2}{\gamma_{i}^{2}}+\gamma_{i}^{2}\right) .
$$

Proof. Since the function $\Phi(x)=-\log x$ is both convex and 4-convex on $(0, \infty)$ because $\Phi(x)=1 / x^{2}$ and $\Phi^{\prime \prime \prime}(x)=6 / x^{4}$ are positive for $x>0$, therefore (41) can easily be obtained by putting $\Phi(x)=-\log x$ and $\zeta_{i}=1(i=1,2, \cdots, n)$ in (35).

Corollary 34. Assume that the conditions of Corollary 33 are fulfilled, then

$$
\log n-E_{s}\left(m_{2}\right) \geq \frac{1}{2} \sum_{i=1}^{n} \gamma_{i}\left(n-\frac{1}{\gamma_{i}}\right)^{2}\left(\frac{3 \gamma_{i}}{2 n \gamma_{i}+1}\right)^{2} .
$$


Proof. Utilizing the function $\Phi(x)=-\log x$ and $\zeta_{i}=1(1,2$, $\cdots, n)$ in (36), we obtain (42).

Definition 35. Let $m_{1}=\left(\gamma_{1}, \gamma_{2}, \cdots, \gamma_{n}\right)$ and $m_{2}=\left(\zeta_{1}, \zeta_{2}, \cdots, \zeta_{n}\right)$ be arbitrary probability distributions such that $\gamma_{i}, \zeta_{i}>0$ for all $i \in\{1,2, \cdots, n\}$. Then, the Kullback-Liebler divergence is defined by

$$
D_{k l}\left(m_{1}, m_{2}\right)=\sum_{i=1}^{n} \zeta_{i} \log \left(\frac{\zeta_{i}}{\gamma_{i}}\right)
$$

Corollary 36. Assume that $m_{1}=\left(\gamma_{1}, \gamma_{2}, \cdots, \gamma_{n}\right)$ and $m_{2}=\left(\zeta_{1}\right.$ $\left., \zeta_{2}, \cdots, \zeta_{n}\right)$ are positive probability distributions, then

$$
D_{k l}\left(m_{1}, m_{2}\right) \leq \frac{1}{6} \sum_{i=1}^{n} \gamma_{i}\left(1-\frac{\zeta_{i}}{\gamma_{i}}\right)^{2}\left(2+\frac{\gamma_{i}}{\zeta_{i}}\right)
$$

Proof. The function $\Phi(x)=x \log x$ is convex and 4-convex on $(0, \infty)$ because $\Phi^{\prime \prime}(x)=1 / x>0$ and $\Phi^{\prime \prime \prime}(x)=2 / x^{3}>0$ for all $x \in(0, \infty)$. Thus, using (35) by taking $\Phi(x)=x \log x$, we get (44).

Corollary 37. Let the postulates of Corollary 36 be true. Then

$$
D_{k l}\left(m_{1}, m_{2}\right) \geq \frac{3}{2} \sum_{i=1}^{n} \frac{\left(\gamma_{i}-\zeta_{i}\right)^{2}}{2 \gamma_{i}-\zeta_{i}}
$$

Proof. Substituting $\Phi(x)=x \log x, x>0$ in (36), we acquire (45).

Definition 38. For any positive probability distributions $m_{1}$ $=\left(\gamma_{1}, \gamma_{2}, \cdots, \gamma_{n}\right)$ and $m_{2}=\left(\zeta_{1}, \zeta_{2}, \cdots, \zeta_{n}\right)$, the Bhattacharyya coefficient is defined as

$$
C_{b}\left(m_{1}, m_{2}\right)=\sum_{i=1}^{n} \sqrt{\gamma_{i} \zeta_{i}}
$$

Corollary 39. Assume that $m_{1}=\left(\gamma_{1}, \gamma_{2}, \cdots, \gamma_{n}\right)$ and $m_{2}=\left(\zeta_{1}\right.$ $\left., \zeta_{2}, \cdots, \zeta_{n}\right)$ are positive probability distributions; then

$$
1-C_{b}\left(m_{1}-m_{2}\right) \leq \frac{1}{24} \sum_{i=1}^{n} \gamma_{i}\left(1-\frac{\zeta_{i}}{\gamma_{i}}\right)^{2}\left(2+\left(\frac{\gamma_{i}}{\zeta_{i}}\right)^{3 / 2}\right)
$$

Proof. If $\Phi(x)=-\sqrt{x}, x>0$, then $\Phi^{\prime \prime}(x)=(1 / 4) x^{-3 / 2}$ and $\Phi^{\prime \prime \prime \prime}(x)=(15 / 16) x^{-7 / 2}$. Thus, this shows that both $\Phi^{\prime \prime}$ and $\Phi^{\prime \prime \prime}$ are positive on $(0, \infty)$. Hence, this confirms the convexity as well as 4 -convexity of the function $\Phi(x)=-\sqrt{x}$. Therefore, using (35) by choosing $\Phi(x)=-x$, we obtain (47).
Corollary 40. Suppose that the assumptions of Corollary 39 hold, then

$$
1-C_{b}\left(m_{1}, m_{2}\right) \geq \frac{1}{2} \sum_{i=1}^{n} \frac{\left(\gamma_{i}-\zeta_{i}\right)^{2}}{\gamma_{i}}\left(\frac{3 \gamma_{i}}{2 \gamma_{i}+\zeta_{i}}\right)^{3 / 2}
$$

Proof. Using $\Phi(x)=-\sqrt{x}, x>0$, in (36), we obtain (48).

Remark 41. The analogous form of above discrete forms for different divergences, Shannon entropy and Bhattacharyya coefficient, can easily be obtained by utilizing Theorems 3 and 5 .

\section{Conclusion}

There are extensive literature devoted to the Jensen inequality concerning different refinements, extensions, and improvements. Also, there are many bounds obtained for the Jensen gap which provides many interesting and valuable estimates for the Jensen gap. In this note, we proposed a novel technique of obtaining of some significant estimates for Jensen's gap while utilizing a 4-convex function. We obtained the required estimates for the Jensen gap by utilizing the definition of convex function and the famous Jensen inequality. For the support of our main results, we provided some examples for taking some particular convex functions. We presented some consequences of the main results in which some new important improvements for the Hölder and Hermite-Hadamard inequalities are acquired. Furthermore, for some more consequences of the main results, we obtain several relations for power and quasiarithmetic means. Applications of the main results are discussed in the information theory. These applications give many interesting estimates for several divergences, Bhattacharyya coefficient and Shannon entropy.

\section{Data Availability}

No data were used to support this study.

\section{Conflicts of Interest}

The authors declare that there are no conflicts of interest regarding the publication of this paper.

\section{Authors' Contributions}

All authors contributed equally to writing of this paper. All authors read and approved the final manuscript.

\section{References}

[1] M. Adeel, K. A. Khan, Đ. Pečarić, and J. Pečarić, "Levinson type inequalities for higher order convex functions via AbelGontscharoff interpolation," Advances in Difference Equations, vol. 2019, no. 1, Article ID 430, 2019.

[2] K. Ahmad, M. Adil Khan, S. Khan, A. Ali, and Y. M. Chu, "New estimation of Zipf-Mandelbrot and Shannon entropies via refinements of Jensen's inequality," AIP Advances, vol. 11 , no. 1 , article 015147 , pp. 1-10, 2021. 
[3] C. P. Niculescu and L. E. Persson, "Convex functions and their applications. A contemporary approach," in CMS Books in Mathematics, Springer-Verlage, New York, 2006.

[4] S. I. Butt, M. Klaričić Bakula, and J. Pečarić, "Steffensen-Grüss inequality," Journal of Inequalities and Applications, vol. 15, no. 2 , p. $810,2021$.

[5] N. Siddique, M. Imran, K. A. Khan, and J. Pečarić, "Majorization inequalities via Green functions and Fink's identity with applications to Shannon entropy," Journal of Inequalities and Applications, vol. 2020, no. 1, Article ID 192, 2020.

[6] J. Pečarić, L. E. Persson, and Y. L. Tong, Convex Functions, Partial Ordering and Statistical Applications, Academic Press, 1992.

[7] M. Adeel, K. Ali Khan, Đ. Pečarić, and J. Pečarić, "Estimation of f-divergence and Shannon entropy by Levinson type inequalities for higher-order convex functions via Taylor polynomial," Journal of Mathematical and Computational Science, vol. 21, no. 4, pp. 322-334, 2020.

[8] M. Adil Khan, Đ. Pečaric, and J. Pečarić, "Bounds for Shannon and Zipf-Mandelbrot entropies," Mathematicsl Methods in the Applied Sciences, vol. 40, no. 18, pp. 7316-7322, 2017.

[9] M. J. Cloud, B. C. Drachman, and L. P. Lebedev, Inequalities with Applications to Engineering, Springer, Cham Heidelberg New York Dordrecht London, 2014.

[10] A. Waheed, A. U. Rehman, M. I. Qureshi, F. A. Shah, K. A. Khan, and G. Farid, "On Caputo $k$-fractional derivatives and associated inequalities," IEEE Access, vol. 7, pp. 32137-32145, 2019.

[11] L. Horváth, Đ. Pečarić, and J. Pečarić, "Estimations of f- and Rényi divergences by using a cyclic refinement of the Jensen's inequality," Bulletin of the Malaysian Mathematical Sciences Society, vol. 42, no. 3, pp. 933-946, 2019.

[12] S. Leorato, "A refined Jensen's inequality in Hilbert spaces and empirical approximations," Journal of Multivariate Analysis, vol. 100, no. 5, pp. 1044-1060, 2009.

[13] Q. Lin, “Jensen inequality for superlinear expectations," Statistics \& Probability Letters, vol. 151, pp. 79-83, 2019.

[14] S. I. Butt, N. Mehmood, Đ. Pečarić, and J. Pečarić, "New bounds for Shannon, relative and Mandelbrot entropies via Abel-Gontscharoff interpolating polynomial," Mathematical Inequalities \& Applications, vol. 22, no. 4, pp. 1283-1301, 2019.

[15] M. Sababheh, “Improved Jensen's inequality," Mathematical Inequalities \& Applications, vol. 20, no. 2, pp. 389-403, 2017.

[16] Z.-G. Xiao, H. M. Srivastava, and Z. H. Zhang, "Further refinements of the Jensen inequalities based upon samples with repetitions," Mathematical and Computer Modelling, vol. 51, no. 5-6, pp. 592-600, 2010.

[17] M. Adil Khan, S. Khan, and Y. Chu, "A new bound for the Jensen gap with applications in information theory," IEEE Access, vol. 8, pp. 98001-98008, 2020.

[18] D. Costarelli and R. Spigler, "How sharp is the Jensen inequality?," Journal of Inequalities and Applications, vol. 2015, no. 1, Article ID 69, 2015. 